\title{
Efeito do Sexo e do Peso ao Abate sobre a Produção de Carne de Cordeiro. Morfometria da Carcaça, Pesos dos Cortes, Composição Tecidual e Componentes Não Constituintes da Carcaça
}

\author{
Edson Ramos de Siqueira ${ }^{1}$, Christian Deodato Simões ${ }^{2}$, Simone Fernandes ${ }^{3}$
}

\begin{abstract}
RESUMO - Neste experimento, avaliou-se o efeito de sexo (macho e fêmea) e de quatro distintos pesos de abate (28, 32, 36, e $40 \mathrm{~kg}$ ) sobre a morfometria da carcaça, os pesos dos cortes, a composição tecidual e os componentes não constituintes da carcaça, em cordeiros mestiços Ile de France x Corriedale, terminados em confinamento. Utilizaram-se 40 animais (20 machos e 20 fêmeas), desmamados aos 60 dias e alimentados, à vontade, com uma ração com 16,46\% de PB e 67,63\% de NDT. Foram divididos em quatro grupos de machos (G1M, G2M, G3M e G4M), sacrificados, respectivamente, com os pesos supracitados, e, da mesma forma, quatro grupos de fêmeas (G1F, G2F, G3F e G4F). Determinaram-se as medidas da carcaça e, de forma subjetiva, a condição corporal, o grau de conformação e o grau de gordura de cobertura. Calcularam-se os índices de compacidade da carcaça e da perna. Foram registrados os pesos e as porcentagens de sete cortes da meia carcaça, bem como as porcentagens de osso, músculo e tecido adiposo, avaliadas por meio de dissecção do lombo. A espessura da gordura de cobertura foi determinada na região lombar. O estudo morfométrico confirmou maior alongamento ósseo dos machos. De maneira geral, as carcaças apresentaram-se com bom acabamento, segundo os índices de compacidade verificados. Os cortes das carcaças das fêmeas foram mais pesados que os dos machos (exceto para o peso de $28 \mathrm{~kg}$ ao abate), principalmente em função dos maiores teores de gordura. Destaca-se a representabilidade dos pesos da pele e do conteúdo gastrintestinal na determinação do rendimento da carcaça. Pelos resultados obtidos, recomenda-se $28 \mathrm{~kg}$ como peso referência para sacrifício.
\end{abstract}

Palavras-chave: carne de cordeiro, carcaça ovina, ovino, produção de cordeiro

\section{Sex and Slaughter Weight Effects on Meat Production of Lambs. Carcass Morphometric Evaluation, Cuts Weights, Tissues and Offals Percentages}

\begin{abstract}
This experiment was carried out to evaluate the effects of sex (males $\mathrm{x}$ females) and four slaughter weights (28, 32, 36 and $40 \mathrm{~kg}$ ), on carcass morphometry, cuts weights, tissues and offals percentages, from crossbred lambs Ile de France x Corriedale, finished in feedlot. Fourty lambs (20 males and 20 females), were weaned at 60 days of age and were full fed a diet with $16.46 \% \mathrm{CP}$ and $67.63 \%$ TDN. They were divided in 4 male groups (G1M, G2M, G3M and G4M), slaughtered respectively with the mentioned weights, and, in the same way, 4 female groups (G1F, G2F, G3F and G4F). It was determined the carcass measures, body score, carcass conformation and fat grades; carcass and leg compacity index; the weights and porcentages of 7 cuts from the half carcass; muscle, bone and fat tissue percentages from the loin and fat thickness on the loin. The morfometric study showed a higher osseous elongation in the males carcasses. Generally, the compacity index indicated a good carcass finishing. The female carcass cuts were heavier than the male cuts (except for the live weight of $28 \mathrm{~kg}$ ), ascribed to the higher fat content. Among the offals, skin and gastrointestinal content weights showed to be highly representative in the carcass yield determination. Finally, it is recommended the slaughter weight of $28 \mathrm{~kg}$.
\end{abstract}

Key Words: lamb carcass, lamb meat, lamb offals, lamb production, sheep

\section{Introdução}

Os modernos sistemas de produção de cordeiros devem enfatizar, concomitantemente, os aspectos econômicos e qualitativos da carne.

O peso ideal ao sacrifício, um dos principais determinantes da qualidade, é aquele em que a proporção de músculos na carcaça é máxima e a gordura, suficiente para conceder à carne, propriedades senso- riais adequadas à preferência do mercado consumidor (OSÓRIO, 1992). Por outro lado, há que se considerar também a eficiência de conversão alimentar do cordeiro, que diminui, à medida que o peso vivo aumenta.

Vários fatores podem afetar a composição da carcaça, sobretudo a alimentação, o sexo e o genótipo. O cruzamento de carneiros da raça Ile de France com ovelhas Corriedale foi determinado, por PILAR et al. (1993), como efetivo na diminuição da

\footnotetext{
1 Prof. Adjunto do DPEA- FMVZ/ UNESP- Botucatu/SP. CEP 18618-000. Autor para correspondência. Bolsista do CNPq. E.mail: ersiqueira@fca.unesp.br

2 Acadêmico do curso de Zootecnia da FMVZ/ UNESP- Botucatu/SP. Bolsista de iniciação Científica da FAPESP.

3 Zootecnista - DPEA - FMVZ/ UNESP- Botucatu/ SP
} 
espessura de gordura $(2,7 \mathrm{~mm}$ nos cordeiros Corriedale; $1,2 \mathrm{~mm}$ nos mestiços).

Já o efeito de sexo na qualidade da carcaça foi constatado por muitos autores, entre eles COLOMER-ROCHER e ESPEJO (1972), que, estudando mestiços Manchega x Rasa Aragonesa, concluíram ser $26 \mathrm{~kg}$ o peso ótimo ao sacrifício para fêmeas e $30 \mathrm{~kg}$ para machos.

O presente estudo foi conduzido com o objetivo de avaliar o efeito de sexo (macho e fêmea) e de quatro distintos pesos de abate $(28,32,36$ e $40 \mathrm{~kg})$ sobre a morfometria da carcaça, peso dos cortes, composição tecidual e componentes não constituintes da carcaça, em cordeiros mestiços Ile de France $\mathrm{x}$ Corriedale.

\section{Material e Métodos}

Utilizaram-se 20 cordeiros machos inteiros e 20 fêmeas, F1 Ile de France x Corriedale, desmamados aos 60 dias de idade, com $12 \mathrm{~kg}$ de peso médio e oriundos de partos simples. A recria e terminação ocorreram em sistema de confinamento total, com dieta composta por $16,46 \%$ de PB e 67,63\% de NDT, oferecida à vontade.

Por sorteio, os cordeiros foram divididos em quatro grupos de machos (G1M, G2M, G3M e G4M) e quatro de fêmeas (G1F, G2F, G3F e G4F), com cinco animais cada um. Os cordeiros machos e fêmeas, dos grupos 1, 2, 3 e 4, foram sacrificados aos $28,32,36$ e $40 \mathrm{~kg}$ de peso vivo, respectivamente.

Após permanência por 24 horas, em câmara de resfriamento a $4^{\circ} \mathrm{C}$, as carcaças foram medidas, com as articulações tarso metatarsianas mantidas a uma distância de $17 \mathrm{~cm}$, por meio de gancho próprio.

Foram obtidas as seguintes medidas:

- comprimento da perna $(\mathrm{CP})$ : distância entre o períneo e o bordo anterior da superfície articular tarso metatarsiana;

- comprimento interno da carcaça (CIC): distância máxima entre o bordo anterior da sínfise ísquiopubiana e o bordo anterior da primeira costela, em seu ponto médio;

- comprimento externo da carcaça (CEC): distância entre a base da cauda e a base do pescoço;

- largura da garupa (LG): largura máxima entre os trocânteres de ambos os fêmures;

- perímetro da garupa (PG): perímetro desta região anatômica, tomando como referência os trocânteres de ambos os fêmures;

- profundidade do tórax (PT): distância máxima entre o esterno e o dorso da carcaça.

As carcaças foram avaliadas subjetivamente, tendo sido consideradas as seguintes variáveis:

- condição corporal (CC): obtida por palpação da região lombar, atribuindo-se notas de 1 a 5 , sendo 1 para a pior e 5 para a melhor;

- grau de conformação (GC): avaliação visual da carcaça, considerando-a como um todo em relação à espessura de seus planos musculares e adiposos, conferindo-se valor 1 para a conformação mais deficiente e 5, para a excelente.

- grau de gordura (GG): determinado mediante apreciação visual da camada de gordura de cobertura, também numa escala de 1 a 5 , sendo o valor 1 para a excessivamente magra $\mathrm{e} 5$, para a excessivamente gorda.

Foram calculados os índices de compacidade da carcaça, definidos pela relação entre o peso da carcaça fria e o comprimento interno da carcaça e de compacidade da perna, obtido por intermédio da relação entre a largura da garupa e o comprimento da perna.

As carcaças foram divididas ao meio, tendo sido a parte esquerda cortada em sete regiões anatômicas (COLOMER-ROCHER e ESPEJO, 1972), as quais foram pesadas individualmente e calculadas as porcentagens em relação ao todo. Separou-se o lombo para dissecção e determinação dos níveis de músculo, tecido adiposo e osso, tendo sido efetuada neste corte, também, a mensuração da espessura da gordura de cobertura.

Foi efetuada a análise de variância, estudando-se o efeito do peso ao sacrifício e do sexo sobre as variáveis consideradas. O contraste de médias foi realizado pelo teste de Tukey. As análises foram realizadas pelo programa Statistical Analysis System (SAS, 1986).

\section{Resultados e Discussão}

A morfometria da carcaça permite avaliar a conformação de maneira objetiva. Os resultados morfométricos obtidos neste experimento encontram-se na Tabela 1.

Constataram-se efeitos de peso ao sacrifício e de sexo, tanto para o comprimento interno como externo da carcaça. O G2M e G3M foram similares para ambas as variáveis. Entretanto, tanto o G2M como o G2F tiveram comprimentos de carcaça superiores a G1M e G1F, bem como G4M e G4F, em relação a G3M e G3F $(\mathrm{P}<0,05)$.

Para o comprimento da perna, não houve efeito de peso ao abate, tendo havido, porém, efeito de sexo, 
Rev. bras. zootec.

Tabela 1 - Médias e desvios-padrão das medidas da carcaça $(\mathrm{cm})$ : comprimento da perna $(\mathrm{CP})$, comprimento interno $(\mathrm{CIC})$ e externo (CEC) da carcaça, largura da garupa (LG) e perímetro torácico (PT), em machos (M) e fêmeas (F) abatidos aos 28 kg (G1), 32 kg (G2), 36 kg (G3) e 40 kg (G4)

Table 1 - Means and standard deviations for carcass measurements (cm): leg length (LL), carcass internal length (CIL), carcass external lenght (CEL), hindquarters width (HW), ribs rack width (RRW) and thorax depth (TD) of males and females slaughtered at $28 \mathrm{~kg}$ (G1), $32 \mathrm{~kg}$ (G2), $36 \mathrm{~kg}$ (G3) and $40 \mathrm{~kg}$ (G4)

\begin{tabular}{|c|c|c|c|c|c|}
\hline & $\begin{array}{l}\text { Variáveis } \\
\text { Variables }\end{array}$ & Gl & $\mathrm{G} 2$ & $\mathrm{G}$ & $\mathrm{GA}$ \\
\hline $\mathrm{M}$ & $\mathrm{CP}$ & $30,12 \pm 0,89^{\mathrm{Aa}}$ & $31,08 \pm 0,89^{\mathrm{Aa}}$ & $30,76 \pm 0,89^{\mathrm{Aa}}$ & $31,20 \pm 0,89^{\mathrm{Aa}}$ \\
\hline$M$ & $L L$ & & & & \\
\hline $\mathrm{F}$ & $\mathrm{CP}$ & $28,37 \pm 0,10^{\mathrm{Ab}}$ & $26,68 \pm 0,89^{\mathrm{Ab}}$ & $29,92 \pm 0,10^{\mathrm{Aa}}$ & $28,00 \pm 0,89^{\mathrm{Ab}}$ \\
\hline$F$ & $L L$ & & & & \\
\hline M & CIC & $44,32 \pm 0,74 \mathrm{Ca}$ & $47,90 \pm 0,74^{\mathrm{Ba}}$ & $47,96 \pm 0,74^{\mathrm{Ba}}$ & $49,78 \pm 0,74^{\mathrm{Aa}}$ \\
\hline$M$ & $C I L$ & & & & \\
\hline $\mathrm{F}$ & CIC & $43,65 \pm 0,82^{\mathrm{Cb}}$ & $44,54 \pm 0,74^{\mathrm{Bb}}$ & $44,17 \pm 0,82^{\mathrm{Bb}}$ & $47,70 \pm 0,74^{\mathrm{Ab}}$ \\
\hline$F$ & $C I L$ & & & & \\
\hline M & $\mathrm{CEC}$ & $51,32 \pm 1,07 \mathrm{Ca}$ & $54,70 \pm 1,07 \mathrm{Ba}$ & $54,50 \pm 1,07^{\mathrm{Ba}}$ & $59,20 \pm 1,07^{\mathrm{Aa}}$ \\
\hline$M$ & $C E L$ & & & & \\
\hline $\mathrm{F}$ & $\mathrm{CEC}$ & $49,57 \pm 1,20^{\mathrm{Ca}}$ & $53,66 \pm 1,07^{\mathrm{Aa}}$ & $52,47 \pm 1,20^{\mathrm{Bb}}$ & $53,75 \pm 1,07^{\mathrm{Ab}}$ \\
\hline$F$ & $C E L$ & & & & \\
\hline M & LG & $14,36 \pm 0,53^{\mathrm{Ca}}$ & $15,34 \pm 0,53^{\mathrm{Bb}}$ & $16,06 \pm 0,53^{\mathrm{Ab}}$ & $16,50 \pm 0,53^{\mathrm{Ab}}$ \\
\hline$M$ & $H W$ & & & & \\
\hline $\mathrm{F}$ & LG & $14,65 \pm 0,60^{\mathrm{Da}}$ & $16,20 \pm 0,53^{\mathrm{Ca}}$ & $17,97 \pm 0,6^{\mathrm{Ba}}$ & $20,62 \pm 0,53^{\mathrm{Aa}}$ \\
\hline$F$ & $H W$ & & & & \\
\hline M & PT & $23,46 \pm 0,34 \mathrm{Ca}$ & $24,32 \pm 0,34^{\mathrm{Ba}}$ & $24,82 \pm 0,34^{\mathrm{Ba}}$ & $25,92 \pm 0,34^{\mathrm{Aa}}$ \\
\hline$M$ & $R R W$ & & & & \\
\hline $\mathrm{F}$ & PT & $22,3 \pm 0,38^{\mathrm{Da}}$ & $23,70 \pm 0,34^{\mathrm{Ca}}$ & $24,90 \pm 0,38^{\mathrm{Ba}}$ & $27,34 \pm 0,34^{\mathrm{Aa}}$ \\
\hline$F$ & $R R W$ & & & & \\
\hline M & PG & $51,96 \pm 0,98^{\mathrm{Ca}}$ & $55,12 \pm 0,98 \mathrm{Ba}$ & $55,66 \pm 0,98^{\mathrm{Bb}}$ & $57,42 \pm 0,98^{\mathrm{Ab}}$ \\
\hline$M$ & $T D$ & & & & \\
\hline $\mathrm{F}$ & PG & $51,55 \pm 1,10^{\mathrm{Da}}$ & $55,98 \pm 0,08^{\mathrm{Ca}}$ & $59,80 \pm 1,10^{\mathrm{Ba}}$ & $61,80 \pm 0,98^{\mathrm{Ab}}$ \\
\hline$F$ & $T D$ & & & & \\
\hline
\end{tabular}

Letras distintas (maiúsculas na linha e minúsculas na coluna) indicam diferença significativa $(P<0,05)$.

Different letters (capital letters within a row and small letters in collumns) differ $(P<.05)$ by Tukey test.

com superioridade dos machos em relação às fêmeas, nos G1, G2 e G4. Este resultado já era esperado, em razão de a fisiologia do macho promover taxa de crescimento mais elevada (WYLIE et al., 1997) e, conseqüentemente, maior alongamento ósseo em relação às fêmeas.

Tendo em vista que a diferença entre os grupos, em termos de peso ao abate, foi de apenas $4 \mathrm{~kg}$, estes resultados se contrapõem aos citados por COLOMERROCHER e ESPEJO (1972), que não detectaram diferenças no comprimento da carcaça $(46,7 \mathrm{~cm})$, quando compararam animais abatidos aos $22 \mathrm{e} 30 \mathrm{~kg}$. Quanto ao efeito de sexo, os machos superaram as fêmeas nos quatro pesos ao sacrifício para o comprimento interno, e em dois para o comprimento externo (G3 e G4). Tal constatação também explica-se pelo já referido diferencial fisiológico de crescimento entre o macho e a fêmea.

Sobre as medidas da garupa (largura e perímetro), observou-se efeito de peso. Não houve efeito de sexo para a largura da garupa no G1 e para perímetro em G1 e G2. Nos demais, entretanto, as fêmeas superaram os machos, em virtude da anatomia pélvica ser mais avantajada naquelas. Tal realidade explica-se pela adequação anatômica desta região, ao parto.

O perímetro torácico seguiu a tendência lógica de aumentar com a elevação de peso ao abate, não tendo sido verificado, porém, efeito do sexo.

A relação entre medidas, ou destas com o peso da carcaça, ou com determinadas frações dela, originam os índices de compacidade. Neste estudo, consideraram-se os índices de compacidade da perna e da carcaça (Tabela 2).

A compacidade da carcaça apresentou superioridade nos pesos mais elevados ao sacrifício, tanto em machos como em fêmeas. De maneira geral, os valores obtidos neste trabalho superaram os citados por OSÓRIO(1992), que constatou a média geral de 0,20 para carcaças de nove procedências, e também OSÓRIO et al. (1998), que em cordeiros da raça Corriedale, criados em campo natural e sacrificados aos 150 dias de idade, obtiveram índice de 
Tabela 2 - Médias e desvios-padrão dos índices de compacidade da carcaça (CC) e compacidade da perna (CP), em machos (M) e fêmeas (F) abatidos aos 28 kg (G1), 32 kg (G2), 36 kg (G3) e 40 kg (G4)

Table 2 - Means and standard deviations for carcass compacity index (CCI) and leg compacity index (LCl) of males (M) and females (F) slaughtered at $28 \mathrm{~kg}$ (G1), $32 \mathrm{~kg}$ (G2), $36 \mathrm{~kg}$ (G3) and $40 \mathrm{~kg}$ (G4)

\begin{tabular}{|c|c|c|c|c|c|}
\hline & $\begin{array}{l}\text { Variáveis } \\
\text { Variables }\end{array}$ & G1 & $\mathrm{G} 2$ & $\mathrm{G}$ & $\mathrm{GA}$ \\
\hline M & $\mathrm{CC}$ & $0,26 \pm 0,007^{\mathrm{Ba}}$ & $0,28 \pm 0,007^{\mathrm{Ba}}$ & $0,37 \pm 0,008^{\mathrm{Aa}}$ & $0,33 \pm 0,008^{\mathrm{Aa}}$ \\
\hline$M$ & $C C I$ & & & & \\
\hline $\mathrm{F}$ & $\mathrm{CC}$ & $0,25 \pm 0,008^{\mathrm{Ca}}$ & $0,31 \pm 0,007^{\mathrm{Ba}}$ & $0,31 \pm 0,007^{\mathrm{Aa}}$ & $0,36 \pm 0,007^{\mathrm{Aa}}$ \\
\hline$F$ & $C C I$ & & & & \\
\hline M & $\mathrm{CP}$ & $0,47 \pm 0,03^{\mathrm{Ba}}$ & $0,49 \pm 0,03^{\mathrm{Bb}}$ & $0,60 \pm 0,04^{\mathrm{Aa}}$ & $0,52 \pm 0,036^{\mathrm{Bb}}$ \\
\hline$M$ & $L C I$ & & & & \\
\hline $\mathrm{F}$ & $\mathrm{CP}$ & $0,51 \pm 0,04^{\mathrm{Ca}}$ & $0,61 \pm 0,03^{\mathrm{Ba}}$ & $0,52 \pm 0,03^{\mathrm{Cb}}$ & $0,75 \pm 0,03^{\mathrm{Aa}}$ \\
\hline$F$ & $L C I$ & & & & \\
\hline
\end{tabular}

Letras distintas (maiúsculas na linha e minúsculas na coluna) indicam diferença significativa $(P<0,05)$.

Different letters (capital letters within a row and small letters in collumns) differ $(P<.05)$ by Tukey test.

compacidade de 0,16 , bem como os calculados por FERNANDES e SIQUEIRA (1997) e MACEDO (1998). Tais diferenças devem-se ao genótipo utilizado neste estudo, o qual continha $50 \%$ de genes de Ile de France, e ao sistema intensivo de terminação. Para esta variável, não foi observado efeito de sexo.

O índice de compacidade da perna também aumentou nos pesos de abate superiores, como era esperado. Para o mesmo peso de abate (G2M), os resultados foram similares aos apresentados por FERNANDES e SIQUEIRA (1997) e MACEDO (1998). Em termos gerais, as médias estiveram abaixo das constatadas por OSÓRIO (1992), com exceção de G4F, que se assemelharam. O efeito de sexo, neste caso, foi verificado, com exceção do G1. As fêmeas foram superiores em G2 e G4, ao passo que os machos as superaram em G3. A supremacia das fêmeas é natural, tendo em vista possuírem maior largura de garupa, medida usada no cálculo deste índice. Trata-se de uma característica anatômica associada ao parto, portanto mais avantajada na fêmea.

Constam da Tabela 3 os resultados da avaliação subjetiva do animal vivo e da carcaça. A condição corporal foi similar entre os grupos de machos, tendo havido diferenças, entretanto, no caso das fêmeas, com tendência das mais pesadas terem apresentado maiores valores. Mais uma vez recorre-se à maior probabilidade de deposição de gordura por parte das fêmeas, sobretudo as mais pesadas, como explicação para o constatado. MACEDO (1998) encontrou, para cordeiros confi-

Tabela 3 - Médias e desvios-padrão referentes à avaliação subjetiva da carcaça: grau de conformação (GC), grau de gordura (GG) e condição corporal (CC), em machos (M) e fêmeas (F) abatidos aos 28 kg (G1), 32 kg (G2), 36 kg (G3) e 40 $\mathrm{kg}(\mathrm{G} 4)$

Table 3 - Means and standard deviations for subjective variables: conformation level (CL), fat level (FL) and corporal condition index (CCl), of males (M) and females (F) slaughtered at $28 \mathrm{~kg}$ (G1), $32 \mathrm{~kg}$ (G2), $36 \mathrm{~kg}$ (G3) and $40 \mathrm{~kg}$ (G4)

\begin{tabular}{|c|c|c|c|c|c|}
\hline & $\begin{array}{l}\text { Variáveis } \\
\text { Variables }\end{array}$ & Gl & $\mathrm{G} 2$ & G & $\mathrm{G} 4$ \\
\hline M & $\mathrm{GC}$ & $2,69 \pm 0,12^{\mathrm{Ba}}$ & $3,00 \pm 0,12^{\mathrm{Aa}}$ & $2,80 \pm 0,12^{\mathrm{Ba}}$ & $2,80 \pm 0,12^{\mathrm{Ba}}$ \\
\hline$M$ & $C L$ & & & & \\
\hline $\mathrm{F}$ & $\mathrm{GC}$ & $2,43 \pm 0,13^{\mathrm{Ca}}$ & $2,95 \pm 0,12^{\mathrm{Ba}}$ & $2,93 \pm 0,13^{\mathrm{Ba}}$ & $3,15 \pm 0,12^{\mathrm{Aa}}$ \\
\hline$F$ & $C L$ & & & & \\
\hline M & CG & $1,55 \pm 0,23^{\mathrm{Ca}}$ & $1,75 \pm 0,23^{\mathrm{Ba}}$ & $2,15 \pm 0,23^{\mathrm{Aa}}$ & $2,10 \pm 0,23^{\mathrm{Aa}}$ \\
\hline$M$ & $F L$ & & & & \\
\hline $\mathrm{F}$ & GG & $1,87 \pm 0,25^{\mathrm{Ba}}$ & $1,85 \pm 0,23^{\mathrm{Ba}}$ & $1,87 \pm 0,25^{\mathrm{Ba}}$ & $2,40 \pm 0,23^{\mathrm{Aa}}$ \\
\hline$F$ & $F L$ & & & & \\
\hline M & $\mathrm{CC}$ & $2,80 \pm 0,10^{\mathrm{Aa}}$ & $2,90 \pm 0,10^{\mathrm{Ab}}$ & $2,85 \pm 0,10^{\mathrm{Ab}}$ & $2,95 \pm 0,10^{\mathrm{Ab}}$ \\
\hline$M$ & $C C I$ & & & & \\
\hline $\mathrm{F}$ & $\mathrm{CC}$ & $2,80 \pm 0,11^{\mathrm{Ca}}$ & $3,15 \pm 0,10^{\mathrm{BCa}}$ & $3,37 \pm 0,11^{\mathrm{ABa}}$ & $3,65 \pm 0,10^{\mathrm{Aa}}$ \\
\hline$F$ & $C C I$ & & & & \\
\hline
\end{tabular}

Letras distintas (maiúsculas na linha e minúsculas na coluna) indicam diferença significativa $(P<0,05)$.

Different letters (capital letters within a row and small letters in collumns) differ $(P<.05)$ by Tukey test. 
Rev. bras. zootec.

1303

nados, sacrificados com $32 \mathrm{~kg}$, condição corporal um pouco superior ao G2M $(3,13)$. Todavia, os valores obtidos neste trabalho foram maiores que os citados por FERNANDES e SIQUEIRA(1997) e OSÓRIO et al. (1998).

Tendência parecida foi verificada em fêmeas, para a variável conformação da carcaça, que está estreitamente vinculada à condição corporal. Quanto maior o peso, mais elevada a conformação.

Já a quantidade de gordura de cobertura, elevouse gradualmente com o aumento de peso. MACEDO (1998) e FERNANDES e SIQUEIRA (1997), para pesos de abate similares a $\mathrm{G} 2$, constataram maiores espessuras da capa de gordura de cobertura, também em condições de terminação em confinamento. Sur-

preendentemente, não se obteve efeito de sexo. Esta verificação, no entanto, não permite inferir que o nível de gordura tenha sido similar entre os machos e fêmeas, já que o tecido adiposo de cobertura é apenas um componente do teor total. Além disto, trata-se de uma determinação subjetiva, em situação de níveis de gordura relativamente baixos, fato que pode dificultar a detecção de diferenças, muitas vezes ínfimas.

$\mathrm{O}$ estudo dos rendimentos dos cortes da carcaça tem seus resultados apresentados nas Tabelas 4 (pesos) e 5 (porcentagens).

Quanto aos pesos, os animais abatidos com $36 \mathrm{e}$ $40 \mathrm{~kg}$ (G3 e G4) apresentaram os maiores valores; fato lógico, em decorrência da proporcionalidade do crescimento das distintas regiões da carcaça com o

Tabela 4 - Médias e desvios-padrão dos pesos $(\mathrm{kg})$ dos cortes da meia carcaça. Pesos da $1 / 2$ carcaça, do pescoço, da costela descoberta, da costela, da paleta, dos baixos, do lombo, e do pernil, em machos (M) e fêmeas $(F)$ abatidos aos 28 $\mathrm{kg}(\mathrm{G} 1), 32 \mathrm{~kg}$ (G2), $36 \mathrm{~kg}(\mathrm{G} 3)$ e $40 \mathrm{~kg}$ (G4)

Table 4 - Means and standard deviations for the half carcass cuts weights (kg). Weights of: half carcass, neck, ribs under the shoulder, ribs, shoulder, breast and ribs points, loin and leg of males (M) and females (F) slaughtered at $28 \mathrm{~kg}$ (G1), $32 \mathrm{~kg}$ (G2), $36 \mathrm{~kg}$ (G3) and $40 \mathrm{~kg}$ (G4)

\begin{tabular}{|c|c|c|c|c|c|}
\hline$S$ & $\begin{array}{l}\text { Variáveis } \\
\text { Variables }\end{array}$ & Gl & $\mathrm{Q} 2$ & $\mathrm{G}$ & G4 \\
\hline M & $\begin{array}{l}\text { Meia carcaça } \\
\text { Half carcass }\end{array}$ & $5,77 \pm 0,21^{\mathrm{Da}}$ & $6,86 \pm 0,21^{\mathrm{Cb}}$ & $7,47 \pm 0,21^{\mathrm{Bb}}$ & $8,02 \pm 0,21^{\mathrm{Ab}}$ \\
\hline $\mathrm{F}$ & $\begin{array}{l}\text { Meia carcaça } \\
\text { Half carcass }\end{array}$ & $5,58 \pm 0,24 \mathrm{Cb}$ & $7,25 \pm 0,21^{\mathrm{Ba}}$ & $8,51 \pm 0,24 \mathrm{Aa}$ & $8,31 \pm 0,21 \mathrm{Aa}$ \\
\hline M & $\begin{array}{l}\text { Pescoço } \\
\text { Neck }\end{array}$ & $0,44 \pm 0,04^{\mathrm{Ba}}$ & $0,54 \pm 0,04^{\mathrm{Ab}}$ & $0,57 \pm 0,04^{\mathrm{Aa}}$ & $0,61 \pm 0,04^{\mathrm{Ab}}$ \\
\hline $\mathrm{F}$ & $\begin{array}{l}\text { Pescoço } \\
\text { Neck }\end{array}$ & $0,39 \pm 0,05^{\mathrm{Bb}}$ & $0,65 \pm 0,04^{\mathrm{Aa}}$ & $0,69 \pm 0,05^{\mathrm{Ab}}$ & $0,66 \pm 0,04 \mathrm{Aa}$ \\
\hline M & $\begin{array}{l}\text { Cost. descob. } \\
\text { Ribs under the shoulder }\end{array}$ & $0,32 \pm 0,04^{\mathrm{Ca}}$ & $0,37 \pm 0,04^{\mathrm{Bb}}$ & $0,42 \pm 0,04^{\mathrm{Aa}}$ & $0,48 \pm 0,04^{\mathrm{Ab}}$ \\
\hline $\mathrm{F}$ & $\begin{array}{l}\text { Cost. descob. } \\
\text { Ribs under the shoulder }\end{array}$ & $0,29 \pm 0,04 \mathrm{Cb}$ & $0,44 \pm 0,04^{\mathrm{Ba}}$ & $0,56 \pm 0,04^{\mathrm{Aa}}$ & $0,58 \pm 0,04^{\mathrm{Aa}}$ \\
\hline M & $\begin{array}{l}\text { Costela } \\
\text { Ribs }\end{array}$ & $0,50 \pm 0,06^{\mathrm{Ba}}$ & $0,61 \pm 0,06^{\mathrm{Bb}}$ & $0,80 \pm 0,06^{\mathrm{Ab}}$ & $0,83 \pm 0,06^{\mathrm{Ab}}$ \\
\hline $\mathrm{F}$ & $\begin{array}{l}\text { Costela } \\
\text { Ribs }\end{array}$ & $0,56 \pm 0,06^{\mathrm{Ca}}$ & $0,69 \pm 0,06^{\mathrm{Ba}}$ & $1,03 \pm 0,06^{\mathrm{Aa}}$ & $0,97 \pm 0,06^{\mathrm{Aa}}$ \\
\hline M & $\begin{array}{l}\text { Paleta } \\
\text { Shoulder }\end{array}$ & $1,11 \pm 0,05^{\mathrm{Ba}}$ & $1,42 \pm 0,05^{\mathrm{Aa}}$ & $1,56 \pm 0,05^{\mathrm{Aa}}$ & $1,70 \pm 0,05^{\mathrm{Ab}}$ \\
\hline $\mathrm{F}$ & $\begin{array}{l}\text { Paleta } \\
\text { Shoulder }\end{array}$ & $1,10 \pm 0,06^{\mathrm{Ba}}$ & $1,40 \pm 0,05^{\mathrm{Aa}}$ & $1,50 \pm 0,06^{\mathrm{Aa}}$ & $1,47 \pm 0,05^{\mathrm{Aa}}$ \\
\hline M & $\begin{array}{l}\text { Baixos } \\
\text { Breast and ribs points }\end{array}$ & $0,72 \pm 0,06^{\mathrm{Ba}}$ & $0,85 \pm 0,06^{\mathrm{Aa}}$ & $0,83 \pm 0,06^{\mathrm{Ab}}$ & $0,88 \pm 0,66^{\mathrm{Ab}}$ \\
\hline $\mathrm{F}$ & $\begin{array}{l}\text { Baixos } \\
\text { Breast and ribs points }\end{array}$ & $0,65 \pm 0,07 \mathrm{Ca}$ & $0,82 \pm 0,06^{\mathrm{Ba}}$ & $1,07 \pm 0,07 \mathrm{Aa}$ & $1,04 \pm 0,67 \mathrm{Aa}$ \\
\hline M & $\begin{array}{l}\text { Lombo } \\
\text { Loin }\end{array}$ & $0,58 \pm 0,04^{\mathrm{Bb}}$ & $0,69 \pm 0,04^{\mathrm{Bb}}$ & $0,82 \pm 0,04^{\mathrm{Ab}}$ & $0,79 \pm 0,04^{\mathrm{Ab}}$ \\
\hline $\mathrm{F}$ & $\begin{array}{l}\text { Lombo } \\
\text { Loin }\end{array}$ & $0,62 \pm 0,04^{\mathrm{Ba}}$ & $0,82 \pm 0,04^{\mathrm{Aa}}$ & $0,96 \pm 0,04^{\mathrm{Aa}}$ & $0,97 \pm 0,04^{\mathrm{Aa}}$ \\
\hline M & $\begin{array}{l}\text { Pernil } \\
\text { Leg }\end{array}$ & $2,10 \pm 0,07 \mathrm{Ca}$ & $2,34 \pm 0,07 \mathrm{BCa}$ & $2,40 \pm 0,07^{\mathrm{Ba}}$ & $2,71 \pm 0,04 \mathrm{Aa}$ \\
\hline $\mathrm{F}$ & $\begin{array}{l}\text { Pernil } \\
\text { Leg }\end{array}$ & $1,84 \pm 0,08^{\mathrm{Ca}}$ & $2,23 \pm 0,07^{\mathrm{Ba}}$ & $2,68 \pm 0,08^{\mathrm{Aa}}$ & $2,58 \pm 0,07^{\mathrm{Aa}}$ \\
\hline
\end{tabular}

Letras distintas (maiúsculas na linha e minúsculas na coluna) indicam diferença significativa $(\mathrm{P}<0,05)$.

Different letters (capital letters within a row and small letters in collumns) differ $(P<.05)$ by Tukey test. 
aumento total da massa corporal. Estes dois grupos foram semelhantes entre si para todos os cortes, com exceção do pernil, mais pesado em G4M. De maneira geral, G1 tendeu apresentar os menores pesos dos cortes.

Os resultados deste estudo estiveram muito próximos aos obtidos por FERNANDES (1994) e MACEDO (1998), considerando-se pesos de abate entre 30 e $32 \mathrm{~kg}$.

Houve marcante efeito de sexo para os pesos dos cortes. Em G1, as meias carcaças dos machos foram mais pesadas; todavia, nos demais grupos, as fêmeas foram superiores. Não houve diferença entre machos e fêmeas para o peso do pernil. Por outro lado, os lombos das fêmeas pesaram mais nos 4 grupos de peso ao abate, fato que pode ser explicado pela vantagem anatômica delas, nesta região, em função do parto. De maneira geral, entretanto, as vantagens verificadas, das fêmeas em relação aos machos, se devem à maior deposição de gordura.
Ao se analisarem os resultados relativos às porcentagens dos cortes, verifica-se que houve efeito de peso ao abate apenas para a costela dos machos, que foi mais representativa em G3 e G4, do que em G1 e G2. Genericamente, as proporções constatadas se assemelharam às citadas por OSÓRIO (1992), FERNANDES (1994) e MACEDO (1998).

A característica matemática desta variável reforça a lei da harmonia anatômica (BOCCARD e DUMONT, 1960), indo até mais além, já que ela diz o seguinte: “em carcaças de pesos e quantidades de gordura similares, quase todas as regiões corporais se encontram em proporções semelhantes, qualquer que seja a conformação dos genótipos considerados". Neste trabalho, entretanto, observou-se que, mesmo entre grupos abatidos com distintos pesos, as porcentagens dos cortes não diferiram.

$\mathrm{Na}$ Tabela 6 estão dispostos os resultados das quantidades dos tecidos muscular, ósseo e adiposo e

Tabela 5 - Médias e desvios-padrão das porcentagens (\%) dos cortes da meia carcaça em machos (M) e fêmeas (F) abatidos aos $28 \mathrm{~kg}(\mathrm{G} 1), 32 \mathrm{~kg}(\mathrm{G} 2), 36 \mathrm{~kg}$ (G3) e $40 \mathrm{~kg}$ (G4)

Table 5 - Means and standard deviations of the half carcass cuts percentages (\%) of males (M) and females (F) slaughtered at $28 \mathrm{~kg}(\mathrm{G} 1)$, $32 \mathrm{~kg}$ (G2), $36 \mathrm{~kg}$ (G3) and $40 \mathrm{~kg}$ (G4)

\begin{tabular}{|c|c|c|c|c|c|}
\hline $\begin{array}{l}\text { Sexo } \\
\text { Sex } \\
\end{array}$ & $\begin{array}{l}\text { Variáveis } \\
\text { Variables }\end{array}$ & Gl & $\mathrm{G}$ & $\mathrm{G}$ & G4 \\
\hline $\mathbf{M}$ & $\begin{array}{l}\text { Pescoço } \\
\text { Neck }\end{array}$ & $7,63 \pm 0,58^{\mathrm{Aa}}$ & $7,87 \pm 0,58^{\mathrm{Aa}}$ & $7,70 \pm 0,58^{\mathrm{Aa}}$ & $7,68 \pm 0,58^{\mathrm{Aa}}$ \\
\hline $\mathrm{F}$ & $\begin{array}{l}\text { Pescoço } \\
\text { Neck }\end{array}$ & $7,03 \pm 0,65^{\mathrm{Aa}}$ & $8,95 \pm 0,58^{\mathrm{Aa}}$ & $8,25 \pm 0,65^{\mathrm{Aa}}$ & $8,05 \pm 0,58^{\mathrm{Aa}}$ \\
\hline M & $\begin{array}{l}\text { Cost.desc. } \\
\text { Ribs under the shoulder }\end{array}$ & $5,56 \pm 0,44 \mathrm{Aa}$ & $5,48 \pm 0,44 \mathrm{Aa}$ & $5,64 \pm 0,44 \mathrm{Aa}$ & $6,02 \pm 0,44 \mathrm{Aa}$ \\
\hline $\mathrm{F}$ & $\begin{array}{l}\text { Cost.desc. } \\
\text { Ribs under the shoulder }\end{array}$ & $5,28 \pm 0,49^{\mathrm{Aa}}$ & $6,05 \pm 0,44^{\mathrm{Aa}}$ & $6,25 \pm 0,49 \mathrm{Aa}$ & $7,03 \pm 0,44 \mathrm{Aa}$ \\
\hline M & $\begin{array}{l}\text { Costela } \\
\text { Ribs }\end{array}$ & $8,72 \pm 0,62^{\mathrm{Bb}}$ & $8,89 \pm 0,62^{\mathrm{Ba}}$ & $10,74 \pm 0,62^{\mathrm{Aa}}$ & $10,40 \pm 0,62^{\mathrm{Aa}}$ \\
\hline $\mathrm{F}$ & $\begin{array}{l}\text { Costela } \\
\text { Ribs }\end{array}$ & $10,07 \pm 0,70^{\mathrm{Ba}}$ & $9,63 \pm 0,62^{\mathrm{Ba}}$ & $11,91 \pm 0,70^{\mathrm{Aa}}$ & $11,67 \pm 0,62^{\mathrm{Aa}}$ \\
\hline M & $\begin{array}{l}\text { Paleta } \\
\text { Shoulder }\end{array}$ & $19,40 \pm 0,79^{\mathrm{Ab}}$ & $20,70 \pm 0,79^{\mathrm{Aa}}$ & $20,90 \pm 0,79$ Аa & $21,30 \pm 0,79 \mathrm{Aa}$ \\
\hline $\mathrm{F}$ & $\begin{array}{l}\text { Paleta } \\
\text { Shoulder }\end{array}$ & $20,64 \pm 0,88^{\mathrm{Aa}}$ & $20,66 \pm 0,79 \mathrm{Aa}$ & $17,82 \pm 0,88^{\mathrm{Ab}}$ & $17,77 \pm 0,79 \mathrm{Ab}$ \\
\hline M & $\begin{array}{l}\text { Baixos } \\
\text { Breast and ribs points }\end{array}$ & $12,40 \pm 0,75^{\mathrm{Aa}}$ & $12,40 \pm 0,75^{\mathrm{Aa}}$ & $11,12 \pm 0,75^{\mathrm{Aa}}$ & $11,07 \pm 0,75^{\mathrm{Aa}}$ \\
\hline $\mathrm{F}$ & $\begin{array}{l}\text { Baixos } \\
\text { Breast and ribs points }\end{array}$ & $11,70 \pm 0,83^{\mathrm{Aa}}$ & $11,32 \pm 0,70^{\mathrm{Aa}}$ & $12,40 \pm 0,83^{\mathrm{Aa}}$ & $12,50 \pm 0,75^{\mathrm{Aa}}$ \\
\hline M & $\begin{array}{l}\text { Lombo } \\
\text { Loin }\end{array}$ & $10,20 \pm 0,51 \mathrm{Aa}$ & $10,08 \pm 0,51 \mathrm{Aa}$ & $11,00 \pm 0,51^{\mathrm{Aa}}$ & $9,90 \pm 0,51^{\mathrm{Ab}}$ \\
\hline $\mathrm{F}$ & $\begin{array}{l}\text { Lombo } \\
\text { Loin }\end{array}$ & $11,23 \pm 0,50^{\mathrm{Aa}}$ & $11,50 \pm 0,51^{\mathrm{Aa}}$ & $11,41 \pm 0,50^{\mathrm{Aa}}$ & $11,71 \pm 0,51^{\mathrm{Aa}}$ \\
\hline M & $\begin{array}{l}\text { Pernil } \\
\text { Leg }\end{array}$ & $35,50 \pm 0,84^{\mathrm{Aa}}$ & $34,17 \pm 0,84^{\mathrm{Aa}}$ & $32,84 \pm 0,84 \mathrm{Aa}$ & $33,82 \pm 0,84^{\mathrm{Aa}}$ \\
\hline $\mathrm{F}$ & $\begin{array}{l}\text { Pernil } \\
\text { Leg }\end{array}$ & $32,90 \pm 0,40^{\mathrm{Ab}}$ & $30,80 \pm 0,80^{\mathrm{Ab}}$ & $31,60 \pm 0,94^{\mathrm{Aa}}$ & $31,1 \pm 0,84 \mathrm{Aa}$ \\
\hline
\end{tabular}

Letras distintas (maiúsculas na linha e minúsculas na coluna) indicam diferença significativa $(\mathrm{P}<0,05)$.

Different letters (capital letters within a row and small letters in collumns) differ $(P<.05)$ by Tukey test. 
Rev. bras. zootec.

Tabela 6 - Quantidades (g) dos tecidos muscular, ósseo e adiposo e espessura da gordura de cobertura do lombo de cordeiros machos (M) e fêmeas (F) abatidos aos 28 kg (G1), 32 kg (G2), 36 kg (G3) e 40 kg (G4)

Table 6 - Muscle, bone and fat quantities (g), loin fat cover thickness of males (M) and females (F) slaughtered at $28 \mathrm{~kg}(\mathrm{G1}), 32 \mathrm{~kg}(\mathrm{G} 2)$, $36 \mathrm{~kg}(\mathrm{G} 3)$ and $40 \mathrm{~kg}(\mathrm{G} 4)$

\begin{tabular}{|c|c|c|c|c|c|}
\hline $\begin{array}{l}\text { Sexo } \\
\text { Sex } \\
\end{array}$ & $\begin{array}{l}\text { Variáveis } \\
\text { Variables }\end{array}$ & Gl & $\mathrm{G} 2$ & $\mathrm{G}$ & G4 \\
\hline $\bar{M}$ & $\begin{array}{l}\text { Músc. } \\
\text { Muscles }\end{array}$ & $382,00 \pm 42,96^{\mathrm{Aa}}$ & $402,32 \pm 38,42^{\mathrm{Aa}}$ & $410,87 \pm 38,42^{\mathrm{Aa}}$ & $419,99 \pm 38,42^{\mathrm{Aa}}$ \\
\hline $\mathrm{F}$ & $\begin{array}{l}\text { Músc. } \\
\text { Muscles }\end{array}$ & $300,51 \pm 42,96^{\mathrm{Aa}}$ & $351,22 \pm 38,42^{\mathrm{Aa}}$ & $308,38 \pm 42,96^{\mathrm{Aa}}$ & $320,52 \pm 42,96^{\mathrm{Aa}}$ \\
\hline M & $\begin{array}{l}\text { Ósseo } \\
\text { Bone }\end{array}$ & $112,50 \pm 13,13^{\mathrm{Aa}}$ & $86,06 \pm 11,74^{\mathrm{Aa}}$ & $128,04 \pm 11,74^{\mathrm{Aa}}$ & $129,72 \pm 11,74^{\mathrm{Aa}}$ \\
\hline $\mathrm{F}$ & $\begin{array}{l}\text { Ósseo } \\
\text { Bone }\end{array}$ & $119,20 \pm 13,13^{\mathrm{Aa}}$ & $134,71 \pm 11,74^{\mathrm{Aa}}$ & $141,69 \pm 13,13^{\mathrm{Aa}}$ & $145,62 \pm 11,74^{\mathrm{Aa}}$ \\
\hline M & $\begin{array}{l}\text { Adiposo } \\
\text { Fat }\end{array}$ & $102,25 \pm 27,99^{\mathrm{Ba}}$ & $209,69 \pm 25,03^{\mathrm{Aa}}$ & $158,92 \pm 25,03^{\mathrm{Ab}}$ & $162,32 \pm 25,03^{\mathrm{Ab}}$ \\
\hline $\mathrm{F}$ & $\begin{array}{l}\text { Adiposo } \\
\text { Fat }\end{array}$ & $118,05 \pm 27,99^{\mathrm{Ba}}$ & $220,60 \pm 25,03^{\mathrm{Aa}}$ & $191,17 \pm 27,99^{\mathrm{Aa}}$ & $195,78 \pm 25,03^{\mathrm{Aa}}$ \\
\hline M & $\begin{array}{l}\text { Esp. Gord } \\
\text { Loin fat cover thickness }\end{array}$ & $1,49 \pm 0,22^{\mathrm{Aa}}$ & $2,18 \pm 0,22^{\mathrm{Ba}}$ & $1,57 \pm 0,22^{\mathrm{Ab}}$ & $1,05 \pm 0,35^{\mathrm{Ab}}$ \\
\hline $\mathrm{F}$ & $\begin{array}{l}\text { Esp. Gord } \\
\text { Loin fat cover thickness }\end{array}$ & $1,10 \pm 0,24^{\mathrm{Ca}}$ & $2,70 \pm 0,22^{\mathrm{Ba}}$ & $2,65 \pm 0,35^{\mathrm{Ba}}$ & $5,10 \pm 0,49^{\mathrm{Aa}}$ \\
\hline
\end{tabular}

Letras distintas (maiúsculas na linha e minúsculas na coluna) indicam diferença significativa $(\mathrm{P}<0,05)$.

Different letters (capital letters within a row and small letters in collumns) differ $(P<.05)$ by Tukey test.

a espessura da gordura de cobertura, variáveis relativas ao lombo.

Não houve efeito de peso ao abate, nem de sexo, tanto no que se refere ao músculo, como ao osso, corroborando FERNANDES(1994) no que diz respeito ao tecido muscular, porém, não com o tecido ósseo.

Para o tecido adiposo, observaram-se menores quantidades em G1M e G1F, em relação aos demais grupos, todos semelhantes entre si, como também destacou WOOD et al. (1991).
Nos dois pesos mais elevados (G3 e G4), detectou-se efeito de sexo, tendo as fêmeas acumulado mais gordura que os machos. Esta evidência repetiu-se no caso da espessura da gordura, como já era esperado. Cabe ressaltar, que para esta variável, surpreendeu o maior valor constatado para $\mathrm{G} 2 \mathrm{M}$, em relação a G3M e G4M. Desconhece-se a causa de tal resultado.

A tendência verificada para o peso dos distintos tecidos repetiu-se, quando enfocada as porcentagens por eles representada (Tabela 7). Apenas para o

Tabela 7 - Porcentagem dos tecidos muscular, ósseo e adiposo do lombo de cordeiros machos (M) e fêmeas (F) abatidos aos 28 kg (G1), 32 kg (G2), 36 kg (G3) e 40 kg (G4)

Table 7 - Muscle, bone and fat porcentages, loin fat cover thickness of males (M) and females (F) slaughtered at $28 \mathrm{~kg}$ (G1), $32 \mathrm{~kg}(\mathrm{G} 2)$, $36 \mathrm{~kg}$ (G3) and $40 \mathrm{~kg}$ (G4)

\begin{tabular}{|c|c|c|c|c|c|}
\hline $\begin{array}{l}\text { Sexo } \\
\text { Sex }\end{array}$ & $\begin{array}{l}\text { Variáveis } \\
\text { Variables }\end{array}$ & Gl & G & G & G4 \\
\hline M & $\begin{array}{l}\text { Músc. } \\
\text { Muscles }\end{array}$ & $59,59 \pm 4,49^{\mathrm{Aa}}$ & $53,38 \pm 4,02^{\mathrm{Aa}}$ & $53,03 \pm 4,02^{\mathrm{Aa}}$ & $56,13 \pm 4,02^{\mathrm{Aa}}$ \\
\hline $\mathrm{F}$ & $\begin{array}{l}\text { Músc. } \\
\text { Muscles }\end{array}$ & $52,26 \pm 4,49^{\mathrm{Aa}}$ & $45,94 \pm 4,20^{\mathrm{Aa}}$ & $47,55 \pm 4,49^{\mathrm{Aa}}$ & $50,76 \pm 4,49^{\mathrm{Aa}}$ \\
\hline M & $\begin{array}{l}\text { Ósseo } \\
\text { Bone }\end{array}$ & $18,12 \pm 1,95^{\mathrm{Aa}}$ & $11,63 \pm 1,74^{\mathrm{Aa}}$ & $16,95 \pm 1,74^{\mathrm{Aa}}$ & $16.98 \pm 1,74^{\mathrm{Aa}}$ \\
\hline $\mathrm{F}$ & $\begin{array}{l}\text { Ósseo } \\
\text { Bone }\end{array}$ & $20,77 \pm 1,95^{\mathrm{Aa}}$ & $17,55 \pm 1,74 \mathrm{Aa}$ & $21,66 \pm 1,95^{\mathrm{Aa}}$ & $17,19 \pm 1,74^{\mathrm{Aa}}$ \\
\hline M & $\begin{array}{l}\text { Adiposo } \\
\text { Fat }\end{array}$ & $16,48 \pm 3,43^{\mathrm{Bb}}$ & $26,68 \pm 3,06^{\mathrm{Aa}}$ & $21,82 \pm 3,06^{\mathrm{Ab}}$ & $21,79 \pm 3,06^{\mathrm{Ab}}$ \\
\hline $\mathrm{F}$ & $\begin{array}{l}\text { Adiposo } \\
\text { Fat }\end{array}$ & $20,38 \pm 3,43^{\mathrm{Ba}}$ & $26,89 \pm 3,05^{\mathrm{Aa}}$ & $28,65 \pm 3,06^{\mathrm{Aa}}$ & $29,2 \pm 3,06^{\mathrm{Aa}}$ \\
\hline
\end{tabular}

Letras distintas (maiúsculas na linha e minúsculas na coluna) indicam diferença significativa $(P<0,05)$.

Different letters (capital letters within a row and small letters in collumns) differ $(P<.05)$ by Tukey test. 
tecido adiposo encontrou-se diferença entre os pesos de abate, tendo G1 apresentado os menores teores em relação a G2, G3 e G4, que foram iguais. O efeito de sexo foi encontrado em G1, G3 e G4, com superioridade das fêmeas.

Constam da Tabela 8 os pesos dos componentes não constituintes da carcaça e as porcentagens como médias dos quatro pesos considerados.

Observou-se efeito de peso ao abate para alguns componentes, com o aumento destes, em relação à elevação do primeiro, como era de se esperar. Em termos porcentuais, os valores gerais são compatíveis com aqueles apresentados por LOPEZ (1987) e OSÓRIO (1992).

Destaca-se, para o peso da pele, efeito de sexo nos quatro grupos, tendo as fêmeas superado os machos. Para os cordeiros sacrificados tanto com $24 \mathrm{~kg}$ como com 30 kg, LOPEZ (1987) também verificou tendência de as fêmeas apresentarem maiores porcentagens de pele em relação aos machos.

A constatação do presente estudo pode ser atribuída ao maior peso de lã das fêmeas, considerando-se que, em todos os grupos, demoraram mais tempo para atingir os pesos de abate, comparativamente aos machos $(16,29,43$ e 83 dias a mais, respectivamente para $28,32,36$ e $40 \mathrm{~kg}$ ).

Em G3 e G4, constatou-se influência significativa do sexo para fígado, aparelho respiratório, conteúdo gastrintestinal, patas $(\mathrm{M}>\mathrm{F})$ e rins $(\mathrm{F}>\mathrm{M})$.

$\mathrm{O}$ resultado dos quatro primeiros componentes corroboram LOPEZ (1987), entretanto, no caso dos rins, este autor detectou maior peso, também para os machos. SIERRA (1974) constatou, da mesma forma, maior conteúdo digestivo em machos, tendo salientado ser este um dos fatores que explicam o menor rendimento de

Tabela 8 - Médias, desvios-padrão e porcentagens (valores médios dos quatro pesos de abate) em relação ao peso vivo dos componentes não constituintes da carcaça $(\mathrm{kg})$, em machos e fêmeas dos distintos grupos experimentais

Table 8 - Means and standard deviations and percentages (mean values from four slaughter weights) of the offals in relation to the live weight (kg) of males (M) and females (F) slaughtered at $28 \mathrm{~kg}(\mathrm{G1}), 32 \mathrm{~kg}(\mathrm{G} 2), 36 \mathrm{~kg}(\mathrm{G} 3)$ and $40 \mathrm{~kg}(\mathrm{G} 4)$

\begin{tabular}{|c|c|c|c|c|c|c|}
\hline $\begin{array}{l}\text { Sexo } \\
\text { Sex }\end{array}$ & $\begin{array}{l}\text { Variáveis } \\
\text { Variables }\end{array}$ & Gl & $\mathrm{Q} 2$ & G & G4 & $\%$ \\
\hline $\bar{M}$ & Cabeça (Head) & $1,65 \pm 0,10^{\mathrm{Ca}}$ & $1,72 \pm 0,10^{\mathrm{Ca}}$ & $2,08 \pm 0,10^{\mathrm{Ba}}$ & $2,32 \pm 0,10^{\mathrm{Aa}}$ & 5,72 \\
\hline $\mathrm{F}$ & Cabeça (Head) & $1,88 \pm 0,24^{\mathrm{Ba}}$ & $1,88 \pm 0,10^{\mathrm{Ba}}$ & $1,9 \pm 0,10^{\mathrm{Ba}}$ & $2,40 \pm 0,10^{\mathrm{Aa}}$ & 5,97 \\
\hline M & Sangue (Blood) & $1,04 \pm 0,11^{\mathrm{Ca}}$ & $1,22 \pm 0,11^{\mathrm{Ba}}$ & $1,58 \pm 0,11^{\mathrm{Aa}}$ & $1,50 \pm 0,11^{\mathrm{Aa}}$ & 3,92 \\
\hline $\mathrm{F}$ & Sangue (Blood) & $0,95 \pm 0,12^{\mathrm{Ba}}$ & $0,98 \pm 0,11^{\mathrm{Ba}}$ & $1,15 \pm 0,12^{\mathrm{Ba}}$ & $1,86 \pm 0,11^{\mathrm{Aa}}$ & 3,6 \\
\hline M & Pele (Skin) & $2,96 \pm 0,22^{\mathrm{Dd}}$ & $3,42 \pm 0,22^{\mathrm{Cb}}$ & $4,4 \pm 0,22^{\mathrm{Bb}}$ & $5,16 \pm 0,22^{\mathrm{Ab}}$ & 11,6 \\
\hline $\mathrm{F}$ & Pele (Skin) & $3,47 \pm 0,24^{\mathrm{Da}}$ & $4,46 \pm 0,22 \mathrm{Ca}$ & $5,07 \pm 0,24^{\mathrm{Ba}}$ & $5,46 \pm 0,22^{\mathrm{Aa}}$ & 13,5 \\
\hline M & Fígado (Liver) & $0,52 \pm 0,02^{\mathrm{Ba}}$ & $0,54 \pm 0,02^{\mathrm{Ba}}$ & $0,62 \pm 0,02^{\mathrm{Aa}}$ & $0,66 \pm 0,02^{\mathrm{Aa}}$ & 1,75 \\
\hline $\mathrm{F}$ & Fígado (Liver) & $0,47 \pm 0,03^{\mathrm{Ba}}$ & $0,51 \pm 0,02^{\mathrm{Ba}}$ & $0,49 \pm 0,03^{\mathrm{Bb}}$ & $0,59 \pm 0,02^{\mathrm{Ab}}$ & 1,55 \\
\hline M & Coração (Heart) & $0,18 \pm 0,01^{\mathrm{Ba}}$ & $0,19 \pm 0,01^{\mathrm{Ba}}$ & $0,22 \pm 0,01^{\mathrm{Aa}}$ & $0,21 \pm 0,01^{\mathrm{Aa}}$ & 0,57 \\
\hline $\mathrm{F}$ & Coração (Heart) & $0,18 \pm 0,01^{\mathrm{Ba}}$ & $0,16 \pm 0,01^{\mathrm{Ba}}$ & $0,19 \pm 0,01^{\mathrm{Ba}}$ & $0,23 \pm 0,01^{\mathrm{Aa}}$ & 0,55 \\
\hline M & Ap.resp. (Respirat. organs) & $0,64 \pm 0,06^{\mathrm{Aa}}$ & $0,67 \pm 0,67^{\mathrm{Aa}}$ & $0,79 \pm 0,06^{\mathrm{Aa}}$ & $0,83 \pm 0,06^{\mathrm{Aa}}$ & 2,17 \\
\hline $\mathrm{F}$ & Ap.resp. (Respirat. organs) & $0,55 \pm 0,07^{\mathrm{Ca}}$ & $0,65 \pm 0,06^{\mathrm{Aa}}$ & $0,57 \pm 0,07 \mathrm{Aa}$ & $0,63 \pm 0,06^{\mathrm{Ab}}$ & 1,8 \\
\hline M & Baço (Dull) & $0,04 \pm 0,001^{\mathrm{Aa}}$ & $0,04 \pm 0,06^{\mathrm{Aa}}$ & $0,05 \pm 0,06^{\mathrm{Ab}}$ & $0,06 \pm 0,006^{\mathrm{Aa}}$ & 0,14 \\
\hline $\mathrm{F}$ & Baço (Dull) & $0,06 \pm 0,006^{\mathrm{Aa}}$ & $0,05 \pm 0,006^{\mathrm{Aa}}$ & $0,06 \pm 0,006^{\mathrm{Aa}}$ & $0,06 \pm 0,006^{\mathrm{Aa}}$ & 0,17 \\
\hline M & Ap.urin. (Urinary organs) & $0,21 \pm 0,03 \mathrm{Aa}$ & $0,22 \pm 0,03^{\mathrm{Aa}}$ & $0,20 \pm 0,03^{\mathrm{Aa}}$ & $0,23 \pm 0,03^{\mathrm{Aa}}$ & 0,64 \\
\hline $\mathrm{F}$ & Ap.urin. (Urinary organs) & $0,17 \pm 0,04^{\mathrm{Ca}}$ & $0,21 \pm 0,03^{\mathrm{Aa}}$ & $0,28 \pm 0,04^{\mathrm{Aa}}$ & $0,23 \pm 0,03^{\mathrm{Aa}}$ & 0,66 \\
\hline M & Rins (Kidney) & $0,17 \pm 0,03^{\mathrm{Ba}}$ & $0,16 \pm 0,03^{\mathrm{Ba}}$ & $0,21 \pm 0,03^{\mathrm{Ab}}$ & $0,22 \pm 0,03^{\mathrm{Ab}}$ & 0,56 \\
\hline $\mathrm{F}$ & Rins (Kidney) & $0,19 \pm 0,03^{\mathrm{Ca}}$ & $0,20 \pm 0,03^{\mathrm{Ca}}$ & $0,30 \pm 0,03^{\mathrm{Ba}}$ & $0,32 \pm 0,03^{\mathrm{Aa}}$ & 0,73 \\
\hline $\mathrm{M}$ & Estômago (Stomach) & $1,04 \pm 0,15^{\mathrm{Ba}}$ & $0,97 \pm 0,15^{\mathrm{Ba}}$ & $1,39 \pm 0,12^{\mathrm{Aa}}$ & $1,33 \pm 0,15^{\mathrm{Aa}}$ & 3,47 \\
\hline $\mathrm{F}$ & Estômago (Stomach) & $1,25 \pm 0,16^{\mathrm{Ba}}$ & $1,16 \pm 0,15^{\mathrm{Ba}}$ & $1,08 \pm 0,16^{\mathrm{Ba}}$ & $1,78 \pm 0,15^{\mathrm{Aa}}$ & 3,9 \\
\hline M & Intestino (Intestine) & $1,38 \pm 0,16^{\mathrm{Aa}}$ & $1,48 \pm 0,16^{\mathrm{Aa}}$ & $1,56 \pm 0,16^{\mathrm{Aa}}$ & $1,59 \pm 0,16^{\mathrm{Aa}}$ & 4,45 \\
\hline $\mathrm{F}$ & Intestino (Intestine) & $1,32 \pm 0,18^{\mathrm{Aa}}$ & $1,41 \pm 0,16^{\mathrm{Aa}}$ & $1,64 \pm 0,18^{\mathrm{Aa}}$ & $1,81 \pm 0,16^{\mathrm{Aa}}$ & 4,55 \\
\hline M & $\begin{array}{l}\text { Conteúdo gastrint. } \\
\text { Gastrointestinal content }\end{array}$ & $3,97 \pm 0,30^{\mathrm{Aa}}$ & $4,31 \pm 0,30^{\mathrm{Aa}}$ & $4,7 \pm 0,30^{\mathrm{Aa}}$ & $5,11 \pm 0,30 \mathrm{Aa}$ & 13,4 \\
\hline $\mathrm{F}$ & $\begin{array}{l}\text { Conteúdo gastrint. } \\
\text { Gastrointestinal content }\end{array}$ & $3,34 \pm 0,34^{\mathrm{Aa}}$ & $4,25 \pm 0,30^{\mathrm{Aa}}$ & $4,04 \pm 0,34^{\mathrm{Ab}}$ & $3,52 \pm 0,30^{\mathrm{Ab}}$ & 11,3 \\
\hline M & Patas (Foot) & $0,65 \pm 0,05^{\mathrm{Ca}}$ & $0,72 \pm 0,05^{\mathrm{BCa}}$ & $0,79 \pm 0,05^{\mathrm{Ba}}$ & $0,93 \pm 0,05^{\mathrm{Aa}}$ & 2,27 \\
\hline $\mathrm{F}$ & Patas (Foot) & $0,71 \pm 0,06^{\mathrm{Aa}}$ & $0,68 \pm 0,05^{\mathrm{Aa}}$ & $0,54 \pm 0,06^{\mathrm{Bb}}$ & $0,76 \pm 0,05^{\mathrm{Ab}}$ & 2,0 \\
\hline M & Rabo (Tail) & $0,15 \pm 0,02^{\mathrm{Bb}}$ & $0,18 \pm 0,02^{\mathrm{Bb}}$ & $0,24 \pm 0,02^{\mathrm{Aa}}$ & $0,29 \pm 0,02^{\mathrm{Aa}}$ & 0,62 \\
\hline $\mathrm{F}$ & Rabo (Tail) & $0,24 \pm 0,03^{\mathrm{Ba}}$ & $0,26 \pm 0,02^{\mathrm{Ba}}$ & $0,29 \pm 0,03^{\mathrm{Ba}}$ & $0,36 \pm 0,02^{\mathrm{Aa}}$ & 0,84 \\
\hline
\end{tabular}

Letras distintas (maiúsculas na linha e minúsculas na coluna) indicam diferença significativa $(P<0,05)$.

Different letters (capital letters within a row and small letters in collumns) differ $(P<.05)$ by Tukey test. 
carcaça em relação às fêmeas.

A amplitude de variação do rendimento da carcaça ovina é relativamente elevada. Em levantamento bibliográfico efetuado por OSÓRIO (1992), encontraram-se valores de 30,4 a 60,8\%.

Muitos são os fatores determinantes desta variável, porém, analisando-se os resultados deste estudo, concernentes aos componentes não constituintes da carcaça, observa-se a importante participação, sobretudo da pele e do conteúdo gastrintestinal. Estes dois componentes, além de representarem um significativo valor numérico (a soma das médias gerais de ambos implicou em $25 \%$ do peso vivo ao abate), sofrem substancial oscilação: a pele em função das diferentes densidades e diâmetros de fibra e altura das mechas; o conteúdo gastrintestinal, pelos distintos alimentos, com velocidade de passagem variáveis, períodos de jejum nem sempre padronizados, entre outros.

Em verdade, uma mera análise numérica do rendimento, sem levar em consideração as características dos componentes não constituintes da carcaça, não é indicadora da real qualidade desta.

\section{Conclusões}

O estudo morfométrico das carcaças confirmou maior alongamento ós seo dos machos em relação às fêmeas.

Os índices de compacidade obtidos indicam boa massa de tecido muscular e adiposo nas carcaças.

Os cortes das carcaças das fêmeas sacrificadas com 32,36 , e $40 \mathrm{~kg}$ foram mais pesados que os dos machos, principalmente em função dos maiores teores de gordura.

Confirmou-se a lei da harmonia anatômica, considerando-se a similaridade entre os grupos, quanto às porcentagens dos cortes.

Os menores níveis de gordura no lombo foram obtidos nas carcaças dos cordeiros abatidos com $28 \mathrm{~kg}$, tanto machos como fêmeas, sendo este o peso recomendado para o sacrifício dos animais.

As fêmeas apresentaram, genericamente, maiores teores de tecido adiposo do que os machos.

O estudo dos componentes não constituintes da carcaça destacou a representabilidade dos pesos da pele e do conteúdo gastrintestinal na determinação do rendimento. Por outro lado, as peles das fêmeas foram mais pesadas que as dos machos, ao passo que estes apresentaram maior conteúdo gastrintestinal, nos dois maiores pesos ao abate.

\section{Referências Bibliográficas}

BOCCARD , R., DUMONT, B.L. 1960. Etude de la production de la viande chez les ovins. II variation de l'importance relative des differents régions corporelles de l'agneau de boucherie. Annales de Zootechnie, 9(4):355-365.

COLOMER-ROCHER, F., ESPEJO, M. 1972. Determinación del peso óptimo de sacrificio de los corderos procedentes del cruzamiento Manchego x Rasa Aragonesa en función del sexo. Inf. Téc Econ. Agraria, 6:219- 235.

FERNANDES, S. Peso vivo ao abate e características da carcaça de cordeiros da raça Corriedale e mestiços Ile de France $x$ Corriedale, recriados em confinamento. Botucatu/ SP: UNESP, 1994. 82p Dissertação (Mestrado em Zootecnia), Universidade Estadual Paulista, 1994.

FERNANDES, S., SIQUEIRA, E.R. 1997. Efeito do genótipo sobre as medidas objetivas e subjetivas da carcaça de cordeiros terminados em confinamento. Rev. Vet. e Zootec., 9:173-186.

LOPEZ, M. Calidad de la canal y de la carne en los tipos lechal, ternasco y cordero de la raza Lacha y estudio de su desarrollo. Zaragoza/Espanha: Facultad de Veterinaria, 1987. 465p. Tese(Doutorado em Zootecnia) - Universidade de Zaragoza, Zaragoza, 1987.

MACEDO, F.A.F. Desempenho e características de carcaças de cordeiros Corriedale e mestiços Bergamácia x Corriedale e Hampshire Down $x$ Corriedale, terminados em pastagem e confinamento. Botucatu/ SP: UNESP, 1998.72 p.Tese (Doutorado em Zootecnia)- Universidade Estadual Paulista, 1998.

OSÓRIO, J.C.S. Estudio de la calidad de canales comercializadas en el tipo ternasco segun la procedencia: bases para la mejora de dicha calidad en Brasil. Zaragoza, Espanha, 1992. 335p. Tese (Doutorado em Produção Animal)- Universidade de Zaragoza, 1992.

OSÓRIO, J.C. MARÍA, G.A., JARDIM, P. et al. 1998. Caracteres de crecimiento, sacrificio y canal en corderos de raza Corriedale criados en un sistema sostenible sobre pastos naturales de Rio Grande do Sul(Brasil). Inf. Téc.Econ.Agraria, 94(1):63-73.

PILAR, R.C, CASSOL, C., RESTLE, J. et al. 1993. Desempenho e características de carcaças ovinas confinadas. Inf. Cerro Coroado, 5:8-9.

SAS Institute Inc. SAS User'guide: Statistics, SAS Inst. Inc., Cary, North Caroline, 1986.

SIERRA, I. 1974. Resultados iniciales del cruce Romanov x Rasa Aragonesa. Instituto de Economia y Producciones Ganaderas del Ebro(I.E.P.G.E). p.9. Boletim Técnico, 7.

WOOD, J.D., McFIE, H.J.H., POMEROY, R.W. et al. 1980. Carcass composition in four sheep breeds: the importance of type of breed and stage of maturity. Anim. Prod., 30:135-152.

WYLIE, A.R.G., CHESTNUTT, D.M.B., KILPATRICK, D.J. 1997. Growth and carcass characteristcs of heavy slaugther weight lambs: effects of sire breed and sex lamb and relationships to serum metabolites and IGF-1. J. Anim. Sci., 64:309-318.

Recebido em: 21/08/00

Aceito em: 19/04/01 